\title{
Multiple Framework QoE Model based Energy Efficient Transmission Strategy in LTE Networks
}

\author{
Ge Zhihui ${ }^{1}$, Tang Liping ${ }^{1}$, Li Taoshen ${ }^{1}$ and Li Qinghua ${ }^{2}$ \\ ${ }^{1}$ School of Computer, Electronics and Information, Guangxi University, Guangxi \\ Nanning, China \\ ${ }^{2}$ Computer Department, Lishui University, Lishui 323000 China \\ E-mail: gezhihui @foxmail.com
}

\begin{abstract}
Wireless technology is continuously evolving, and the development of new versions of adaptive protocols and mechanisms are at their peak to achieve high performance in network-based applications and services. LTE networks offer broadband wireless access to users who can benefit from high data rate applications such as video streaming. However, the high energy consumption of these applications has not been considered. The end user quality of experience (QoE) of video delivery over a radio network is mainly influenced by the radio parameters in the radio access network. This paper will present a multiple framework QoE model for video delivery over LTE, denoted as MQoE that measures the overall perceived quality of mobile video delivery from subjective user experience and objective system performance. We propose a QoE-based energy efficient mechanism to reduce smartphones' power consumption. We have done a series of experiments to verify the efficiency of this model. Experiment results show that MQoE has significant improvement at energy efficiency
\end{abstract}

Keywords: QoE; energy efficiency; LTE network

\section{Introduction}

LTE, an abbreviation for Long-Term Evolution, commonly marketed as 4G LTE, is a standard for wireless communication of high-speed data for mobile phones and data terminals. In LTE network, TCP dominates the transport-layer protocol usage on mobile devices and HTTP in application layer [1]. Researchers pay their attentions to the video delivery optimization for fairly maximizing the video clients' QoE by ignoring the devices power consumption. Intelligent mobile terminal as a power limited device, the power consumption is very high in long stream transmission. It has been an urgent to develop a strategy to save energy. Researchers are paying their attentions to power characteristic of LTE network [4]. In this paper, we try to give a tradeoff between QoE and energy efficiency.

The QoE of smartphone applications and services perceived by users depends on many factors including anomalies in the network, application, and also the energy consumption. Applications that run on mobile devices, especially video streaming applications, accumulate significant amounts of data. Challenge for operators is to manage operational costs, while keeping the end-user's perceived quality high enough. Our challenge is to minimize energy consumption without significantly compromising user experience.

The rest of this paper is organized as follows: In Section II we present the related work in this area. In Section III we analyze users QoE used the proposed QoE model MQoE. In Section IV we describe the proposed MQoE mechanism in detail. In Section V we present the performance evaluation results. Finally, in Section VI we give the conclusions of this work. 


\section{Related Work}

The energy efficiency of LTE network has been widely studied in recent years. Ref [2] gives an in-depth study of LTE network protocol and application performance. The authors found that the TCP flow's performance was very low. According to analysis of TCP performance [3], Huang proposed a bandwidth estimation algorithm and found that bandwidth utilization was often lower than $50 \%$.This LTE-unfriendly behavior leads to a large cost in the process of data download and was detrimental for both QoE and power of terminal equipments. In [4], researchers develop the first empirically derived comprehensive power model of a commercial LTE network, which accurately models UE energy usage. Ickin $[5,6]$ pointed that network interface was one of the main energy consumption modules of mobile devices. For the needs of real-time update of content transmission, the network module requires constant switch to receive or transmit data. Investigations show that limiting the network traffic and increasing the resource utilization efficiency amongst the applications and services can highly reduce the total energy consumption. Ickin developed an application called ExpCO2, which could guarantee the QoE and minimize energy consumption. However, this application only applied to the user didn't interact with mobile phone and some applications based on cloud services. Studies show that when QoE is poor, 65\% of users will try another access technology, 22\% choose to give up, 13\% choose to try again. Modeling QoE is challenging due to the difficulties in representing a complex subjective measure of user experience in a simple and objective way. International standards for measuring QoE are MOS and E-model [7]. The E-model (Recommendation G.107)[8] predicts the quality affected by various transmission impairments of bandwidth, delay, jitter and loss. Wei [9] analyzed the user acceptability. Based on the acceptability data obtained from user studies on quality acceptance assessment of mobile device, this paper concentrates on the quality acceptability-based QoE (A-QoE) modeling. Reference-free QoE models rely on seeking the factors that cause the quality loss in the entire video delivery process. In [10], the researchers propose adaptive Discontinuous Reception (DRX) parameters for LTE that reduce the energy consumption of mobile devices without degrading the video quality of adaptive HTTP streaming users. In this work, we determine adaptive DRX parameters for multiple adaptive HTTP streaming sessions in a QoE optimized LTE system in order to reduce the average power of the UEs' radio interface without lowering the visual quality of the video and thus make the QoE-driven system more energy-efficient. However, this method should determine the relationship between QoE and transmission rate, and the DRX parameters are chosen according to transmission constraints and adapted periodically to the varying conditions in order to ensure the overall MOS maximization. So that mobile devices need to constantly communicate with the base station to update DRX parameters, which increase the communication time and power consumption. Video streaming applications run on mobile devices always carry amounts of data. The success of video applications running via radio networks depends on the video QoE perceived by the end-users [11]. There have been efforts to relate the network level measurements to user-perceived voice quality [12-13]. However, research on user-perceived video QoE modeling is still limited [14]. Objective metrics for perceptual video quality assessment (VQA) are often used as objective QoE (oQoE) in video services [15]. These models focus on the impact of low-level video characteristics on human visual system (HVS) and are developed to fit in MOS gained from subjective assessments. The QoE of smartphone applications and services perceived by users depends on many factors including anomalies in the network, application, and also the energy consumption. At network-level, high packet delay variation causes long video freezes that 
eventually impact negatively the end-user perceived quality. The freezes can be quantified as large time gaps in-between the displayed pictures during a video stream at the application-level. All above studies motivate our study to develop QoE models to estimate user-perceived quality and to save energy.

\section{MQoE Model}

We establish an analytic model called MQoE, which contains objective and subjective evaluation methods for QoE. We use MOS and Survival Rate (SR) to evaluate QoE. In evaluation of subjective QoE factors, user's perception is represented by numerical values. Table 1 depicts a single stimulus Absolute Category Rating (ACR) method with fivegrade quality scale, e.g., Mean Opinion Score (MOS). The user-perceived QoE is translated into numbers ranging from 1 to 5. MOS and SR are used in objective and subjective assessment of the user-perceived QoE throughout the paper.

\section{Table 1. ITU-T Scale of Media Quality Impairment MOS Table}

\begin{tabular}{|c|c|c|c|c|c|}
\hline Scale & 5 & 4 & 3 & 2 & 1 \\
\hline Quality & Excellent & Good & Fair & Poor & Bad \\
\hline
\end{tabular}

\subsection{Framework of MQoE}

QoE has evolved over the years from end-to-end QoS due to the QoS was not powerful enough to express the quality in a communication service. ITU-Telecommunication Standardization Sector (ITU-T) E.800 [20] defines QoS as "the collective effect of service performance, which is relying on individual technical measures e.g., Key Performance Indicators (KPI).

The existing QoE diagnosis is often performed based on network-level measurements with technical metrics such as bandwidth, packet loss, packet delay, or packet delay variation. However, these only technical metrics based QoE models are limitations to evaluate user-perceived quality. Therefore, user-centric QoE has the role of networkcentric QoS. QoE increases the importance of user-perceived quality instead of technical performance in the overall service, and tries to achieve adding human's hedonic and aesthetic needs to the evaluation of user perception. So, we establish a solid, theoretical and practical framework for QoE evaluation model. We call it MQoE model. MQoE is based on objective and subjective measures. Figure 1 is the framework of MQoE. Our study attempts to investigate QoE on video streaming Apps at network level and application level. At the same time we take user cognition and user behavior into consideration. In this framework, the MQoE model contains 1) two-levels QoS metrics: network QoS (NQoS) and application QoS (AQoS) as well as 2) two-level QoE metrics: user cognition QoE (CQoE) and user behavior QoE (BQoE). This model combines QoS with QoE, which will be described in the next. 


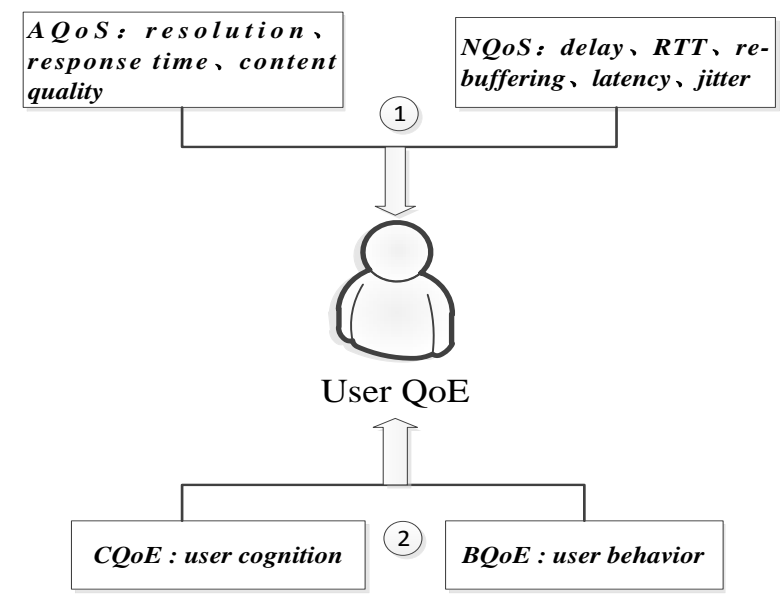

Figure 1. The MQoE Framework

\subsection{The Relationship between QoS and QoE}

The next important QoE modeling step is to map the relationship between the QoE and the variable. We propose a simple method to establish the relationship between QoE and several QoS mechanism. First, we quantify the QoE-QoS correlation for each QoS by analyzing technical metrics, and the mapping relationship can be derived as the following equations:

$$
\left\{\begin{array}{l}
Q o E=f_{Q o s_{1} \rightarrow Q o E}\left(Q o S_{1}\right) \\
Q o E=f_{Q o s_{2} \rightarrow Q o E}\left(Q o S_{2}\right) \\
\cdots \ldots \\
Q o E=f_{Q o s_{n} \rightarrow Q o E}\left(Q o S_{n}\right)
\end{array}\right.
$$

Noted that $\operatorname{QoS}_{1} \cdots \operatorname{QoS}_{\mathrm{n}}$ in this study represent different QoS metrics. We use MOS or Survival Rate (SR) to describe QoE in next. MOS is a subject measurement to obtain the human user's view of the quality, which is the arithmetic mean of individual scores, and range from 1 (worst) to 5 (best). SR is the object measure to record the duration time of users spending on video streaming, which describes user engagement on video playing. We hypothesize users may quit video as they feel unsatisfied or unacceptable during the playing process.

Second, we apply Eq.2 to obtain the multiple level QoE-QoS mapping relationship.

$$
Q o E=\alpha \cdot f_{Q O s_{1} \rightarrow Q 0 E}\left(Q O S_{1}\right) \cdot f_{Q 0 s_{2} \rightarrow Q 0 E}\left(Q O S_{2}\right) \cdot \ldots \cdot f_{Q O s_{n} \rightarrow Q O E}\left(Q o S_{n}\right)
$$

The constant $\alpha$ is estimated by the least square method to minimize the square error of predictions made by this equation. When choosing the QoS metrics in this study, we selected video resolution, re-buffering, and startup delay as metrics.

During the QoS metrics selection phrase, we conduct a Kano model [21] evaluation. The Kano model has been widely practiced in various industrial fields as an effective tool to understand user preferences on service and product quality [22]. It was proposed by professors (Noriaki Kano, et.) at Tokyo University of Technology (ET). It's the first time to introduce the standard of satisfaction and dissatisfaction into the field of quality management.

We combined the Kano model with QoS metrics selection. In our study, we selected video resolution $(R v)$, re-buffering frequency ( $r b f)$, and startup delay (delay) as QoS metrics. So the QoE-QoS mapping relationship is shown in Eq.3. In (3) the x represents the respective factors. 


$$
\left\{\begin{array}{l}
f_{R_{v} \rightarrow \text { MOS }}(x)=1.475 \ln (x)-6.15 \\
f_{r b f \rightarrow M O S}(x)=0.738 e^{-x}+2.266 \\
f_{\text {delay } \rightarrow \text { MOS }}(x)=-0.02 x+2.53
\end{array}\right.
$$

\section{Energy Efficient MQoE}

In this section, we study the QoE from network, application, and energy perspectives. We try to achieve trade-off between QoE and energy efficiency.

According to the selection of QoS metrics and user-perceived metrics, there exits some interaction between QoE and energy consumption. Now we have got two inspirations:

\section{(1) Based on the mapping relationship of QoE-QoS}

Aware and predict user behavior on application layer;

\section{(2) Based on the CQoE and BQoE}

According to user's emotion and behavior to determine whether the current request link is terminated. When requesting video, the MQoE mechanism predicts user behavior according to current link quality. We get users' QoE status by monitoring the link condition. If user feels that QoE is bad, it will trigger an interruption by sending interrupt link order. Media streaming in LTE network, usually, after user dropping out the application, the background process does not turn off the current service. There still exits data transmission in background. Though Http state is closed, the TCP link is still connected. So, when user triggers the interrupt request, they should exit application and close the link to increase the sleeping time of the device and reduce energy consumption of equipment at the same time.

Algorithm 1: INIT initialization parameters for the algorithm which include network information, i.e. network identification, bandwidth, etc. $T_{d}$ is a delay timer for data arrival. $\alpha$ is a delay value within the scope of user acceptance. link is the current link that user request. $s r$ is a reflect of the CQoE and BQoE. random_No is a random number which is range from 0 to 1 .

\begin{tabular}{|c|c|c|}
\hline & & Algorithm 1 TSE (INIT, link, $T_{d}, \alpha, s r$, random_No $)$ \\
\hline & & $1 \quad$ if $(I N I T=$ TRUE $)$ then \\
\hline & & init_f(sr); \\
\hline & & init_P( $\alpha)$; \\
\hline & & Start(link); \\
\hline & & SendMulticast(ExpNewInVideo,f); \\
\hline & & ListenLink_Info $\left(b d, T_{d}\right)$; \\
\hline & & if $\left(T_{d}>\alpha\right)$ then \\
\hline & & QoE damaged; \\
\hline & & Break(link); \\
\hline & & Stop(link); \\
\hline & & else \\
\hline & & Continue(link); \\
\hline & & end if: \\
\hline & & if(random_No $<s r)$ then \\
\hline & & Break(link); \\
\hline & & Stop (link); \\
\hline & & end if; \\
\hline & & FinishMuticast(ExpNewInVideo); \\
\hline & & Closed(link); \\
\hline & & return $f$ \\
\hline
\end{tabular}




\section{Evaluation}

We evaluate the performance of MQoE energy efficiency by using experiments in our indoor testbed with real environment, shown in Figure 9. We compare MQoE to default mechanism in these experiments. All evaluations are carried out on ZTE U9180 with Android platform version 4.3, which has a standard $3.7 \mathrm{~V} \mathrm{Li-ion}$ battery. The network type is TD-LTE. We use 10 devices in our first experiment. It reflected power changes by measuring electric current.

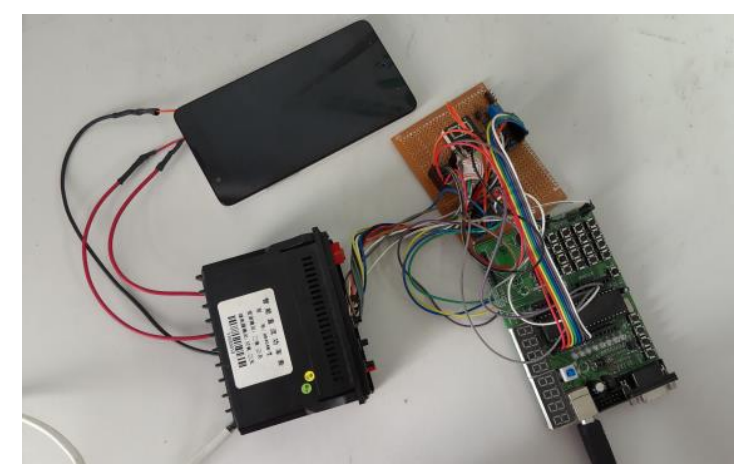

Figure 9. Experiment and Measurement uses ZTE U9180

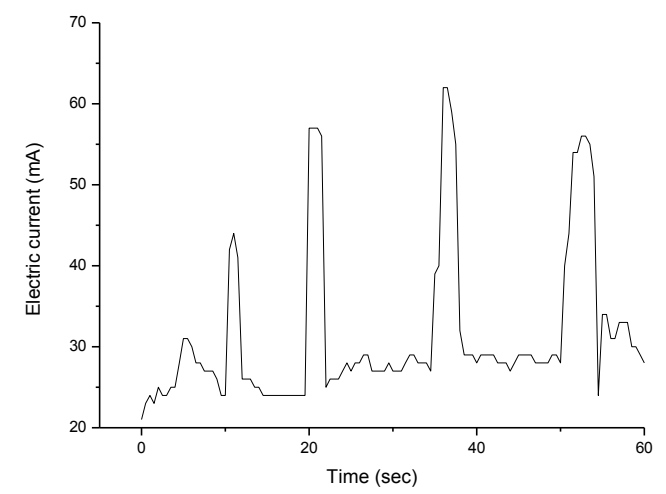

\section{Figure 10. Electric Current Changes in Traditional Mechanism}

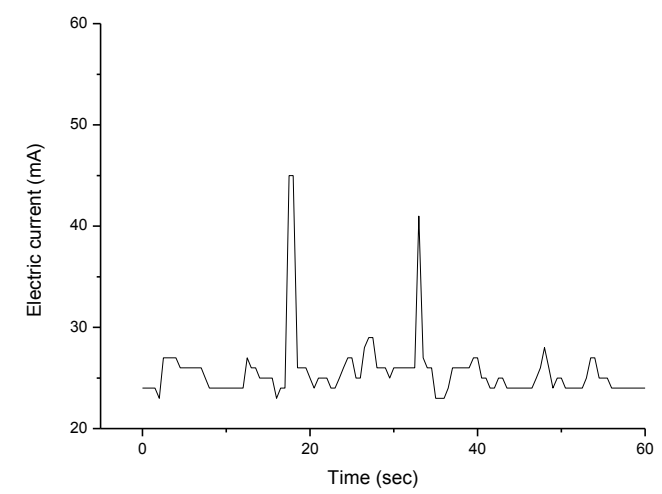

Figure 11. Electric Current Changes in MQoE Mechanism

We used the same video in both parts; the streamed video content was 60s. Figure 10 and Figure 11 show the traditional mechanism and MQoE mechanism of the current changes. In Figure 10, there were several delays in transmission with a higher current curve, which happened in 10s, 20s, 35s and 45s. In Figure 11, we installed the MQoE energy efficient mechanism on the test video application, its current change is gentle, and the change is not obvious. In Figure 11 we can find that in the last 20 seconds, the gap of the peak is larger than the first 40 s, then the gap changes smaller. This is because in LTE network sending data packets has higher synchronization rate prone to transient network congestion, especially the wireless network is easier to lead packets loss. Random delay is to solve network congestion problems in the common method. After running for a while user felt the delay is too long. QoE might be damaged and tried to stop the request.

Use this device to compare the two mechanisms' power consumption based on NQoS and AQoS. From Figure 12, we compared the energy consumption when the delay was $7 \mathrm{~s} 、 8 \mathrm{~s} 、 10 \mathrm{~s}$. We can find that the traditional mechanism obviously increases its energy consumption, and the energy consumption increases very fast. Using MQoE mechanism, energy changes smoothly, and the energy consumption increases slowly. We also took a 
test to compare the energy consumption based on BQoE and CQoE, as shown in Figure 13, and the result was similar with Figure 12. We can conclude from the above experiments, that MQoE has more significant effect.

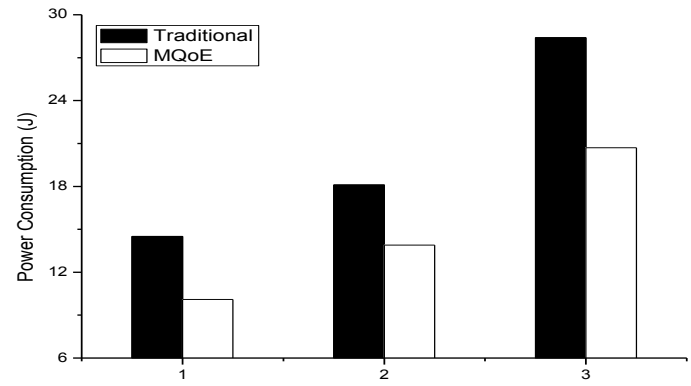

Figure 12. Compare the Power Consumption based on QoE-QoS

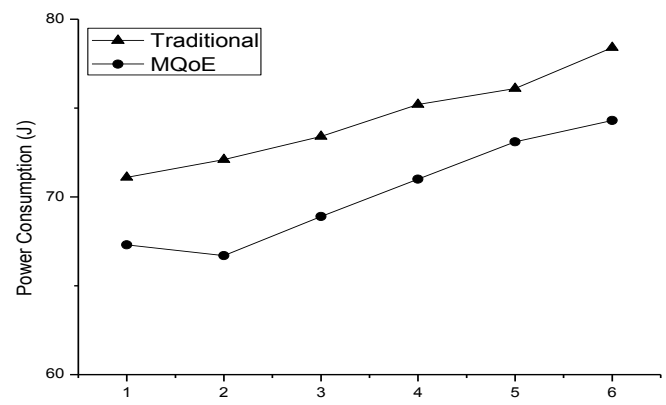

Figure 13. Compare the Power Consumption based on BQoECQOE

\section{Conclusion}

The QoE focusing on video streaming application on smartphones has been studied in this paper. The MQoE model involves both objective and subjective measurements conducted via android smartphone. This ideal QoE model is efficient, lightweight and suitable for video content. Thus it can be easily to analyze the userperceived quality. In this paper, we present a mechanism of energy-efficiency on LTE network: MQoE mechanism. By theoretical analysis we found the main factors of user QoE. By estimating the current network environment and analyzing the power consumption, we have given the reasonable strategy to achieve energy saving effect. Real environment experimental results show the effectiveness of MQoE mechanism. Devices power consumption can be reduced about 10-30\%.

\section{Acknowledgements}

This work is supported by the National Natural Science Foundation of China (Grant No 61363067, 60963022) and Scientific research project Y201432644 of Zhejiang Provincial Education Department.

\section{References}

[1] Q. Xu, J. Erman and A. Gerber, "Identifying diverse usage behaviors of smartphone apps", Proceedings of the ACM SIGCOMM conference on Internet measurement conference, (2011).

[2] J. Huang, F. Qian and Y. Guo, "An in-depth study of LTE: Effect of network protocol and application behavior on performance", Proceedings of the ACM SIGCOMM 2013 conference on SIGCOMM, (2013).

[3] V. Jacobson, R. Braden and D. Borman, “TCP Extensions for High Performance”, RFC 1323, (1992).

[4] J. Huang, F. Qian and A. Gerber, "A close examination of performance and power characteristics of 4G LTE networks", Proceedings of the 10th international conference on Mobile systems, applications, and services, (2012).

[5] S. Ickin, K. Wac and M. Fiedler, "QoE-based energy reduction by controlling the $3 \mathrm{~g}$ cellular data traffic on the smartphone", Proceedings of Energy Efficient and Green Networking (SSEEGN), 22nd ITC Specialist Seminar Christchurch, (2013).

[6] T. Han, N. Ansari and M. Wu, "On accelerating content delivery in mobile networks", Communications Surveys \& Tutorials, vol. 15, no. 1314, (2013).

[7] W. W. Cheng, "The study of accessment methods for the quality of user experience on wireless acceess system”, Xi'an: Xi'an University of Science and Technology, (2013).

[8] I. G. Rec, "107-The E Model, a computational model for use in transmission planning", International Telecommunication Union, vol. 8, no. 20, (2003).

[9] W. Song and D. W. Tjondronegoro, "Acceptability-based QoE models for mobile video", Proceedings of the IEEE Transactions on Multimedia, vol. 16, no. 738, (2014). 
[10] S. Khan, D. Schroeder and A. El Essaili, "Energy-efficient and QoE-driven adaptive HTTP streaming over LTE", Proceedings of the Wireless Communications and Networking Conference (WCNC), (2014).

[11] A. Khan, L. Sun and E. Ifeachor, "QoE prediction model and its application in video quality adaptation over UMTS networks", IEEE Transactions on Multimedia, vol. 14, no. 431, (2013).

[12] S. R. Quackenbush, T. P. Barnwell and M. A, "Clements. Objective measures of speech quality", Prentice Hall, (1988)

[13] W. D. Voiers, "Diagnostic acceptability measure for speech communication systems", Proceedings of the Acoustics, Speech, and Signal Processing, IEEE International Conference on ICASSP'77, (1977).

[14] C. Lin, J. Hu and X. Z. Kong, "The review about model and evaluation of user quanlity of experience (QoE)", Computers, vol. 35, no. 1, (2012).

[15] T. Zinner, O. Hohlfeld and O. Abboud, "Impact of frame rate and resolution on objective QoE metrics", Proceedings of Second International Workshop on Quality of Multimedia Experience (QoMEX), (2010).

[16] K. Kilkki, "Quality of Experience in Communications Ecosystem", Universal Computer Science, vol. 14, no. $615,(\mathbf{2 0 0 8})$.

[17] ITU-T Report, Definition of Quality of Experience (QoE), (2007).

[18] K. U. R. Laghari and K. Connelly, "Toward total quality of experience: A QoE model in a communication ecosystem", Communications Magazine., vol. 50, (2012), pp. 58-65.

[19] Cisco Visual Networking Index: Global mobile data traffic forecast update, 2011-2016, White Paper, (2012).

[20] ITU-T Rec. E.800, Terms and definitions related to the quality of telecommunication services, (1995).

[21] X. Lai, M. Xie and K. Tan, "Optimizing product design using the kano model and qfd", Proceeding of Engineering Management Conference, (2004).

[22] S. Ickin, K. Wac, M. Fiedler, L. Janowski, J. H. Hong and A. K. Dey, "Factors influencing Quality of Experience of commonly-used mobile applications", IEEE Communications Magazine, vol. 50, no. 48, (2012).

[23] R. K. P. Mok, E. W. W. Chan and R. K. C. Chang, "Measuring the quality of experience of HTTP video streaming", Proceedings of IFIP/IEEE International Symposium on Integrated Network Management (IM), (2011).
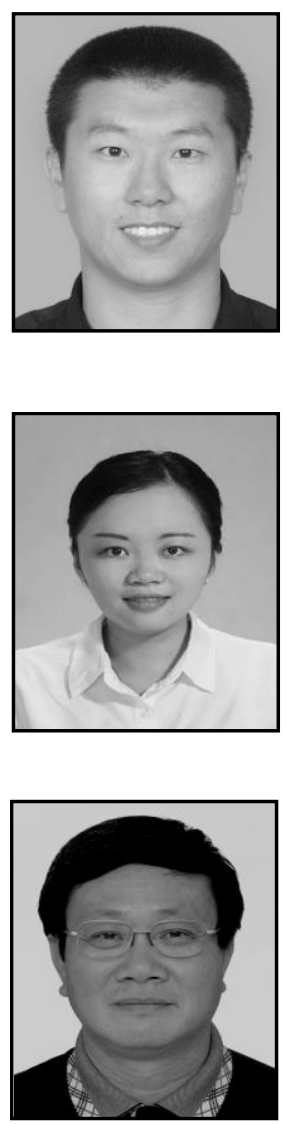

\section{Authors}

Ge Zhihui, he received a B.S. degree in Computer Science from the Beijing Technology and Business University, Beijing, China, in 2001, and a M.S. degree in Computer Science from the Guangxi University, Guangxi, China, in 2004 and a Ph.D. degree in Computer Science from the Central South University, Hunan, China, in 2007. $\mathrm{He}$ is a professor in the Guangxi University.Nanning, Guangxi, China. His research interests are in wireless networks. E-mail: gezhihui @foxmail.com

Tang Liping, she was born in Guangxi, China, in 1990. She received a B.S. degree in Computer Science from the Guangxi University, Guangxi, China, in 2013, and is a M.S. student in Computer Science from the Guangxi University, Guangxi, China. Her research interests are in wireless networks. E-mail: tlp_291314@163.com

Li Taoshen, he received the B.S., M.S. and $\mathrm{PhD}$ degrees in computer science and technology from the Central South University, Changsha, People's Republic of China, in 1982, 1989 and 2008, respectively. He is now a Professor and the Dean of School of Computer, Electronics and Information in Guangxi University, China. His research interests are in the area of distributed database system, wireless mesh netwxorks, cloud computing and intelligent processing. 